\title{
Providing context to acid metalliferous drainage assessments
}

\author{
P.D.S. Rousseau Golder Associates Pty Ltd, Australia
}

\begin{abstract}
The why, what, how, when, where and whom questions with respect to completing an acid metalliferous drainage (AMD) assessment are outlined in this discussion.

Why? AMD has been recognised as the most significant potential long-term liability associated with mining, particularly post closure. The requirement to understand AMD is thus driven by corporate governance, financing and regulatory considerations.

What? The current definition (GARD Guide, 2009; and other guideline documents) of what an AMD assessment should include is broad. It includes acidity that is released through sulphide oxidation, but also covers release of other elements that are mobile under acidic, neutral, or alkaline conditions, as well as introduced contaminants. The purpose of these assessments is thus to identify all mining-related sources of potential contamination.

How? To achieve a realistic AMD assessment, specialist knowledge about geochemistry, hydrogeology, engineering and management details of a project is necessary to evaluate AMD risk, to model systems in question, and to develop mitigation strategies. To evaluate the potential for impacts it is necessary that contaminant sources, transport pathways and receptors be identified and that their physical and geochemical properties must be adequately quantified. These properties must also be related to the mining operation using a suitable site conceptual model.

When? AMD assessment should commence at the exploration stage, once sufficient geological information and sample material is available to obtain a realistic view of ore and waste geochemistry. The earlier the evaluation starts, the sooner AMD related liabilities and technical challenges associated with geochemistry for a mining project become known.

Where? The AMD assessment should consider all materials to be disturbed by mining that could potentially result in significant geochemical changes and associated mobilisation of acidity or other potentially harmful substances.

Whom? An AMD assessment should be carried out by technical specialists, suitably knowledgeable about geochemical and hydrogeological processes. Sufficient geological background is required to conceptualise relevant geological factors associated with a mining operation. It is also necessary for geological, mining and engineering personnel associated with a project to provide input to these assessments, as their information and activities can have a major influence on AMD related outcomes associated with material management.
\end{abstract}

\section{Introduction}

The past ten years have seen a major increase in focus on acid and metalliferous drainage ((AMD), current Australian usage). This phenomenon is the same as acid mine drainage (with the same abbreviation) and acid rock drainage (ARD) as it is referred to internationally. This paper aims to contextualise the reasons for this focus and the mechanisms and reasoning by which AMD assessments should be approached, as well as to summarise relevant aspects of AMD and its assessment. A number of guidelines have been released over the years, most recently Price (2009) by MEND in Canada, the Global Acid Rock Drainage Guide (GARD Guide, 2009) by INAP and Managing Acid and Metalliferous Drainage by DoITR (2007) in Australia. Various research programmes have also concentrated on research into AMD, notably the MEND programme in Canada, ADTI in the United States, MiMi in Sweden, the Water Research Commission in South Africa and various institutions in Australia along with INAP and other industry funded initiatives. These studies and 
guidelines provide a technical framework for practitioners to assess AMD, but often do not communicate the philosophical and technical rationale associated with the recommended approaches.

This paper has been written in response to observations that key personnel (not necessarily AMD practitioners) involved in mining projects, often do not have sufficient understanding of the rationale associated with the assessment and management of AMD. These personnel may, however, be directly or indirectly associated with management of AMD. Practical experience has highlighted a number of factors that are frequently misunderstood or unknown, thus providing the inspiration for this summary.

\section{Why are AMD assessments required?}

Despite AMD assessments being primarily a technical exercise, their greatest impacts are political and financial.

Although AMD has occurred since mining began, the scale of mining and mining methods have changed significantly since the industrial revolution, but especially in the past 50 years with globalisation and the proliferation of open pit mining. Along with increasing world populations the scale of mining has also increased, resulting in significant social and regional environmental impacts in various parts of the world.

AMD has particularly become prominent in the past decade. This is associated with a convergence of factors that have elevated it to the highest rated environmental risk in hard rock mining. The contamination of the Fly River at Ok Tedi in 1999 may be one contributing factor as a case where a real environmental catastrophe contributed to public awareness and action. The reasons that AMD is of such high significance today is, however, more likely related to financial occurrences in the United States (Kirschner and Grandy, 2003).

The depressed metal prices of the 1990s resulted in a number of failed mining companies. In addition to this bonding companies in the U.S. collapsed after the insurance fallout related to the 11 September 2001 terrorist attacks. The combination of these factors left governments with unbonded mining liabilities to contend with (Kirschner and Grandy, 2003). Through this process the actual costs of mine closure became apparent to the regulatory agencies and changes were implemented to the methods by which reclamation bonds were valued.

Reclamation was previously costed based on the surface area that needed to be reclaimed, however, regulators now required that long-term water treatment costs associated with AMD should be included in closure costs as this had not previously been considered. In some cases bonds increased in value by more than 100 fold (Kirschner and Grandy, 2003) to reflect the added costs of perpetual water treatment. These large increases in bond amounts are perhaps the best indicator of the significance that is now attributed to AMD compared with other closure and reclamation liabilities.

Along with the lack of government appetite for mining risk, increasing environmental awareness has resulted in mining environmental legislation being enacted in virtually every country, including low income developing countries. Although this legislation is not equally applied and technical standards are not equal everywhere as far as regulators are concerned, there are also other drivers that influence the assessment of AMD for mining projects.

Most of the major international banks are Equator Principle Financial Institutions (EPFIs). The Equator Principles are a set of 10 principles for project financing applied by signatory financial institutions. They apply to all projects that exceed $\$ 10$ million in value and aim to ensure that lending is only provided to institutions that demonstrate responsible social and environmental stewardship. According to the requirements of the Equator Principles, loans are only provided to projects complying with Principles 1 to 9. The principles are applied to all new projects and expansions of existing projects and deal with management mechanisms to ensure that responsible lending practices are implemented. The Equator Principles are primarily considered to be a mechanism for managing risk. Although social and environmental risks are the primary focus, financial risk to the institutions themselves is also an important consideration. Details are available at www.equator-principles.com.

The viability of project development is further affected by the inclusion of environmental considerations in listing requirements on Canadian stock exchanges, with the Toronto Stock Exchange (TSX) being the most significant. These requirements are outlined in the National Instrument 43-101 standard of disclosure for 
mineral projects, which is applied to all mining projects listed on the TSX in any capacity. It is specifically required that environmental considerations and closure costs are to be disclosed and these aspects can thus affect whether a project is considered viable or not.

Besides the abovementioned external factors, mining companies are increasingly adopting corporate policies that require social and environmental responsibility. Many have implemented internal Environmental Management Systems (EMSs) or accredited management systems, in particular ISO 14001 . These systems require that suitable corporate policies be in place and that operations comply with all legal and technical practices required for acceptable environmental performance. As rehabilitation requirements become stricter and mines close, mining companies have also realised retrospectively that better planning and operation of closed mines would have prevented or reduced large liabilities they now face. This has led to increasing inclusion of environmental considerations in planning and operation of mines and associated corporate policies, guidelines and staffing.

Environmental awareness amongst the general population has vastly increased since it came to prominence in the 1970s. There is thus a significant resistance to projects that are seen to be harmful to social or environmental wellbeing, which has only been increased by a number of high profile mining accidents. This translates into political activism and even mainstream resistance to irresponsible development. The increasing popularity of Green parties internationally is a good measure of this aspect.

The requirement for AMD assessments to be carried out is thus driven primarily by risk avoidance by all project stakeholders, since its significance as the primary long-term risk associated with hard rock mining has become apparent.

\section{What should be included in an AMD assessment?}

An AMD risk assessment is intended to identify any materials that may be exposed or created during mining that will result in a long-term environmental geochemical risk. The term has thus become broader reaching in recent times than solely oxidation of sulphide minerals.

The primary reason that mining presents a geochemical risk is because comminution exposes fresh mineral surfaces to oxygen and water. This causes more rapid weathering and release of mobile geochemical species with subsequent transport in contact water. The weathering processes may include oxidation of sulphide minerals but can also includes other weathering processes that break down rock materials and release potentially harmful elements.

Besides sulphide oxidation, mineral solubility or weathering of non-sulphide minerals can also result in the release of a number of potentially toxic elements (metals) into contact waters, although this is strongly influenced by the local geology and climate. It is also worth noting that some elements associated with sulphide minerals can pose a hazard, despite the material in question being classified as non acid generating.

Elements that are not necessarily associated with acid production are potentially mobile at circum neutral or alkaline $\mathrm{pH}$ and include aluminium, arsenic, antimony, boron, cadmium, fluorine, manganese, mercury, molybdenum, selenium, thallium, vanadium and uranium. Many of these elements are particularly sensitive to redox conditions in the waters where they occur. Once rock is exposed, the speciation, i.e. mineralogy and aqueous speciation, is the main control on weathering and transport behaviour, assuming that water is available to transport contaminants so generated.

Typically mines expose various materials that have the potential to cause AMD, including:

- For all mines:

o ore stockpiles

$\circ$ heap leach residues

$\circ$ tailings

- borrow pit materials for infrastructure development, depending on geology

- contact waters from processing or water treatment 
- any other materials or fluids generated from mineral processing or activities associated with mining, e.g. water treatment.

- For open cut mines:

- all waste rock material to be generated from mining, including all exposed lithologies

- exposed materials on pit slopes and potentially in the blast fracturing zone.

- For underground mines:

- shaft excavation materials

$\circ$ underground workings exposures

$\circ$ waste rock (if any is generated).

Besides the inherent geochemical characteristics of a mineral deposit, the potential for contaminants to be transported is the second major factor that will control the potential for impacts to occur. Contaminant transport generally occurs through surface water erosion or groundwater seepage through mineralised materials. It is thus imperative to have a suitably detailed understanding of transport mechanisms, quantities and pathways to understand the potential for harm from a mining operation. Mine waste and stockpile materials may also be transported as dust and this factor should not be ignored in an AMD assessment, as dust can act as an important diffuse source of contamination.

Depending on the level of assessment, it may also be necessary to identify potential receptors to demonstrate the potential or lack thereof for impacts. By adding this component, the AMD assessment becomes a risk assessment with defined objectives.

\section{$4 \quad$ How should AMD be assessed?}

Three components of a system are generally defined for environmental risk assessments:

1. A contaminant source.

2. A pathway to transport contaminants.

3. A receptor that may be harmed by contamination.

This approach is also used to assess the risk posed by AMD. Understanding the risk posed by AMD requires adequate knowledge of the geochemical and physical (hydrogeological/hydrological, climatic, ecological and social) environment in which a mining project is to be developed.

The geochemical nature of the materials determine whether AMD can be generated and its geochemical characteristics, i.e. what can or will be leached and at what concentrations. The geochemical characteristics, as well as physical factors determine its severity and whether it has the potential to cause harm.

Therefore the first consideration of interest is the geochemical nature of the material. This is also the main topic of this section as it is the linkage that is more often poorly understood and is regularly carried out by non geochemists with a limited geological/mineralogical background. It is also still a source of significant debate.

\subsection{Static geochemical testing}

The general classification of AMD risk is primarily based on static geochemical test methodologies, commencing with acid-base accounting (ABA). An ABA assessment classifies the 'measured' potential for a material to generate a net amount of acidity into risk categories. ABA is initially calculated conservatively as the balance of total sulphur concentration in a sample (as pyrite equivalents) relative to the sample's acid neutralising capacity (by titration).

Another test that is used with increasing frequency is the net acid generation (NAG) test (AMIRA, 2002). This test relies on rapid oxidation of a sample to release all acidity and then allowing this acidity to react with available neutralising capacity in the same sample. Any excess acidity that is generated is then measured by titration with sodium hydroxide (typically using cut-off values of $\mathrm{pH} 4.5$ and $\mathrm{pH} 7$ ). 
The ABA and NAG tests can be further refined and corrected to account for the reactivity and acid generating capacity of different mineral species. The detailed methodologies are documented in various guideline documents, e.g. Price (2009) and AMIRA (2002). The tests will not be discussed in detail here, as it is their application that is of most interest for the purposes of this paper.

Despite the application of different static test methodologies, their ultimate aim is to quantify the total potential for acid generation and the total potential to neutralise any acid that is generated. Where methodologies differ most is in the interpretation of the data that is generated from these tests. Two somewhat different classification approaches have been developed primarily in North America and in Australia for using ABA and NAG test results to classify AMD risk. These methodologies and their implications are summarised hereunder, retaining terminology as used in the respective regions to highlight differences and similarities.

The Canadian standard (Price, 2009; and formerly Price, 1997) is the most frequently used internationally. This methodology reports ABA results in terms of acid potential (AP) and neutralisation potential (NP) in equivalents of $\mathrm{CaCO}_{3}$, whereas the Australian standard reports the same data as maximum potential acidity (MPA) and acid neutralising capacity (ANC) in equivalents of $\mathrm{H}_{2} \mathrm{SO}_{4}$. The quantitative difference between using the different reporting units is only approximately $2 \%$ and thus has very little practical relevance. It should also be noted that AP vs. MPA and NP vs. ANC are effectively the same measurements, although various test methodologies have been used to measure them, generally variations on the original methodology developed by the U.S. Geological Survey (Sobek et al., 1978). The greatest difference between the North American and Australian methodologies is related to the way these results are used for classification.

The North American approach uses both the difference and the ratio of NP to AP to evaluate whether an excess of neutralising potential is available to react with any potential acidity that may be generated. These quantities are expressed as NP-AP or net neutralising potential (NNP) and NP/AP or net potential ratio (NPR). The Australian methodology uses the net acid production potential (NAPP), which is equivalent to NNP, along with NAG test results to classify the risk of acidification of a sample.

The current classification schemes used in Canada (Table 1) and Australia (Table 2) respectively are presented in the following tables: 
Table 1 Sample classification criteria for ABA tests (Price, 2009) as used in Canada

\begin{tabular}{|c|c|c|}
\hline Sample Classification & $\begin{array}{l}\text { Classification } \\
\text { Criterion }\end{array}$ & $\begin{array}{l}\text { Errors That May Occur and Can Affect These } \\
\text { Classifications }\end{array}$ \\
\hline \multirow[t]{4}{*}{$\begin{array}{l}\text { Sample is Potentially } \\
\text { Acid Generating (PAG) }\end{array}$} & NPR $<1$ & $\begin{array}{l}\text { Acid generated from AP is neutralised by sources in } \\
\text { addition to measured NP. }\end{array}$ \\
\hline & & $\begin{array}{l}\text { At very low reaction rates neutralisation capacity of } \\
\text { silicate minerals may be underestimated due to the short } \\
\text { period over which laboratory tests are carried out. }\end{array}$ \\
\hline & & $\begin{array}{l}\text { Sulphur minerals containing the sulphur used to calculate } \\
\text { AP may generate }<2 \text { moles of acid per mole of sulphur. }\end{array}$ \\
\hline & & $\begin{array}{l}\text { NP and AP measurements are made on whole samples of } \\
\text { material with NP preferentially exposed on surfaces, with } \\
\text { AP contained within coarse particles. }\end{array}$ \\
\hline \multirow[t]{4}{*}{ Sample is Non-PAG } & $\mathrm{NPR}>2$ & $\begin{array}{l}\text { NP is depleted by acid produced from other processes than } \\
\text { sulphide mineral oxidation, e.g. acidic flush water. }\end{array}$ \\
\hline & & $\begin{array}{l}\text { NP is less effective at neutralisation than calcite and is } \\
\text { unable to maintain a near-neutral } \mathrm{pH} \text {. }\end{array}$ \\
\hline & & $\begin{array}{l}\text { Sulphide or acid sulphate minerals may generate or release } \\
\text { more than } 2 \text { moles of acid per mole of sulphur. }\end{array}$ \\
\hline & & $\begin{array}{l}\text { NP and AP measurements are made on whole samples of } \\
\text { material with AP preferentially exposed on surfaces, with } \\
\text { NP contained within coarse particles. }\end{array}$ \\
\hline $\begin{array}{l}\text { Sample classification is } \\
\text { uncertain until NPR } \\
\text { criterion is refined }\end{array}$ & $1 \leq \mathrm{NPR} \geq 2$ & $\begin{array}{l}\text { Assuming no errors in the prediction of effective AP and } \\
\text { NP, the maximum NPR capable of generating ARD will be } \\
\text { between } 1 \text { and } 2 \text {. The classification of a sample with an } \\
\text { NPR between } 1 \text { and } 2 \text { may remain 'uncertain' until the } \\
\text { NPR criterion is refined. }\end{array}$ \\
\hline
\end{tabular}

Table 2 Sample classification criteria for ABA tests (DoITR, 2007), as used in Australia

\begin{tabular}{lll}
\hline Primary Geochemical Material Type & NAPP $\left(\mathrm{kg} \mathrm{H}_{2} \mathbf{S O}_{4} / \mathbf{t}\right)$ & NAG $\mathbf{~ p H}$ \\
\hline Potentially Acid Forming (PAF) & $>10^{*}$ & $<4.5$ \\
Potentially Acid Forming-Low Capacity (PAF-LC) & 0 to $10^{*}$ & $<4.5$ \\
Non Acid Forming (NAF) & Negative & $\geq 4.5$ \\
Acid Consuming (ACM) & Less than -100 & $\geq 4.5$ \\
$\quad$ Uncertain\# & Positive & $\geq 4.5$ \\
$\quad$ Uncertain\# & Negative & $<4.5$ \\
\multicolumn{1}{c}{ Uncertain\# } & Positive & $<4.5$ \\
\hline
\end{tabular}

* Site-specific but typically in the range 5 to $20 \mathrm{~kg} \mathrm{H}_{2} \mathrm{SO}_{4} / \mathrm{t}$.

\# Further testing required to confirm material classification.

It is clear from these classification tables that various uncertainties may affect the classification that one obtains from $\mathrm{ABA}$ tests and that professional judgement and further testing is generally a necessity to obtain a reliable indication of AMD potential. There are also considerations inherent to the particular classification schemes that have to be kept in mind. An example is the use of NAPP as opposed to NPR to classify AMD risk in a sample, as demonstrated in Figure 1. 


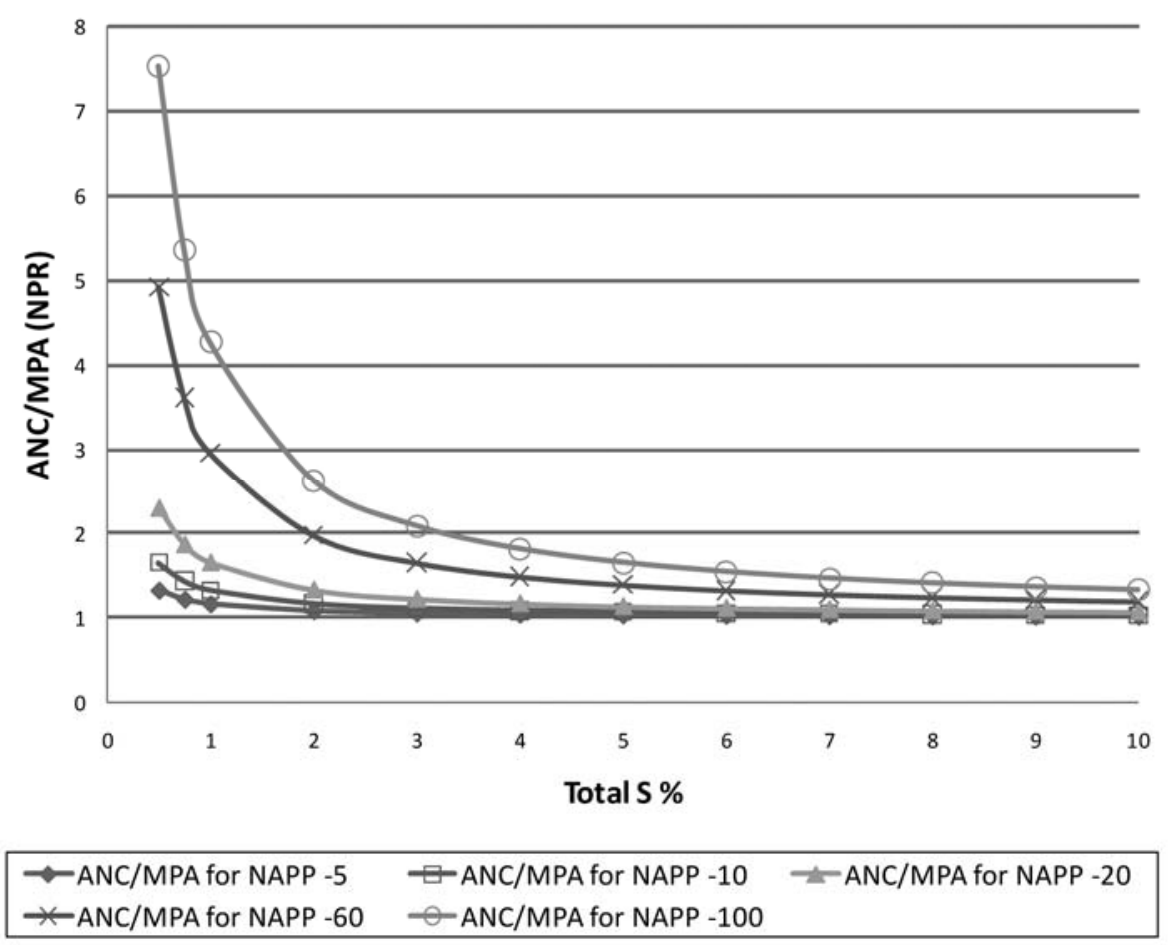

Figure 1 Comparison of NPR (ANC/MPA) and NAPP as a function of total sulphur concentration

It is clear from Figure 1 that the use of these classification schemes is not equal and the following observations are relevant:

- Using the NPR provides an indication of relative excess of neutralising minerals relative to acid producing sulphide minerals. The use of this methodology, however, requires that a large excess of neutralising minerals be present at higher sulphur concentrations to maintain a Non-PAG classification. A number of factors that will be discussed later tend to favour this approach, as it is reasonable to be more conservative at higher sulphur concentrations, if these criteria are indeed conservative.

- The use of NAPP to classify a sample's AMD potential is questionable, as the level of conservatism is strongly influenced by the available acidity in a sample. When comparing different NAPP values between -5 and -100 it can be deduced from Figure 1 that the NPR (ANC / MPA ratio) rapidly drops below 2 at an exponential rate with increasing sulphur concentration. This implies that any samples with an MPA exceeding approximately $3 \% \mathrm{~S}$ (equivalent to about 11 to $12 \%$ pyrite) will have an NPR value of $<2$ according to the Canadian classification, despite having an NAPP value of -100 .

Besides the differences in classification schemes, additional considerations are necessary when assessing static geochemical test data. Besides the uncertainties associated with the test methods themselves (as summarised in Table 1), it is also necessary to understand that the sample classifications obtained using static test methodologies are not necessarily directly transferable to materials at a mine site. Static tests occur in what are effectively closed systems, with the exception of $\mathrm{CO}_{2}$ gas that can escape from reactions between carbonate minerals and acid. The tests thus assume that reactions run to completion.

There are a number of cases where this assumption is not valid and this is probably the rule rather than the exception. Some important considerations in this respect are summarised below and can be understood in the context of Equations (1) and (2):

- Under field conditions it is frequently the case that geochemical systems are not closed and that acidity and alkalinity are released from rock materials and flushed away in surface water and groundwater. This is frequently observed where circum-neutral mine waters (buffered by carbonate 
minerals between $\mathrm{pH} 7$ and $\mathrm{pH}$ 8) drain from mining operations. Where this occurs only half the available alkalinity is used for neutralisation, with the rest being lost in the drainage water.

- In some cases drainage may occur in completely closed systems where gas cannot escape. In this case the neutralisation reactions can also not run to completion as $\mathrm{CO}_{2}$ gas cannot escape. When this occurs neutralisation will also occur at less than optimal rates.

$$
2 \mathrm{CaCO}_{3}(\mathrm{~s})+2 \mathrm{H}^{+} \rightarrow 2 \mathrm{Ca}^{2+}+2 \mathrm{HCO}_{3}^{-}
$$

(Initial dissolution reaction buffered at approx $\mathrm{pH} 8$ )

$$
2 \mathrm{H}^{+}+2 \mathrm{HCO}_{3}^{-} \rightarrow 2 \mathrm{H}_{2} \mathrm{O}+\mathrm{CO}_{2}(\mathrm{~g})
$$

(Bicarbonate reaction with additional acid to $\mathrm{pH} 4.5$ )

In both the above cases the minimum ratio of ANC to MPA or NP to AP that would be required would be 2 to 1 to allow for sufficient neutralising capacity to prevent acidic drainage from developing. This is excluding such considerations as availability of neutralising minerals or possible outside influences, e.g. acidic rainfall.

Besides measuring the quantity of excess of neutralising capacity, the application of static test results should thus adequately account for climatic and hydrological/hydrogeological conditions at a mine site and its facilities as well. This was previously accounted for by applying higher factors of safety, e.g. samples were previously only considered to be Non-PAG at NPR values of $\geq 4$ (Price, 1997). The recent reduction in the 'safe' NPR is thus more likely associated with mining companies finding it difficult and expensive to achieve NPR values of $\geq 4$, rather than a lack of technical merit associated with the original classification. This is also reflected in the stated assumptions associated with the revised classification.

Alongside the potential for acidification, it is also essential to understand the leaching potential of rock components that may be toxic to the environment. As mentioned previously, some of these elements are only mobile under acidic conditions, however, a number of elements are also mobile under circum-neutral or alkaline conditions.

To evaluate such processes, it is necessary to have an adequate understanding of the geochemistry of the materials of interest. This generally implies adequate knowledge of the mineralogical composition of the material, its elemental composition and the leaching potential under conditions that would be relevant and representative under field conditions at a mine site for any material of interest.

Poor characterisation of leaching potential frequently causes errors in AMD assessments as some practitioners evaluate leaching potential based on elemental composition alone, or through the use of 'Standard' test methodologies that have no relevance under field conditions for the materials in question. Although kinetic tests (usually humidity cells) remain the most reliable method to assess leachate characteristics, along with reaction rates, appropriately designed static tests can also provide some insight into leachate characteristics. This, however, requires that expected mineral reactions can be adequately simulated with the chosen leaching solution or that the leachate generated is at least able to provide sufficient data for a conservative assessment of leachate chemistry.

Many of the guideline documents do not provide adequate direction regarding such static leaching tests, although they are discussed to some extent in Price (2009) and to a lesser extent in the GARD Guide (2009). The relevance of leaching is discussed in the DoITR (2007) document, but little guidance is provided regarding method selection. It should be noted, however, that practitioners require sufficient geochemical knowledge to apply professional judgement when specifying and interpreting these tests, as they can cause serious errors of judgement if incorrectly or inappropriately applied.

\subsection{Kinetic testing}

Kinetic testing is applied to test samples in order to measure reaction rates and to attempt to simulate geochemical processes that occur under field conditions. The use of humidity cells is the favoured methodology internationally, although kinetic leaching columns are the standard method used in Australia. 
There are two schools of thought associated with the application of kinetic test data to field conditions, i.e.:

1. Leachates generated from kinetic tests are essentially representative of water quality that will be generated from the materials being tested.

2. Kinetic test results can only be applied to field conditions after appropriate correction for site specific characteristics.

Both of these assumptions can be valid under different conditions and require that the practitioner apply adequate judgement prior to using data for modelling or assessment purposes. Experience has shown that many practitioners apply data without such forethought.

Data that is evaluated according to the first school of thought (point number 1 above) is valid only if leachate compositions are controlled through mineral solubility constraints. This is often the case if secondary minerals control the solution composition. However, it is seldom the case that secondary minerals control the concentrations of all components of a solution, so this methodology can in all likelihood only be applied for particular solutes of interest. If this approach is used, virtually any kinetic test method should produce similar results.

The second method of evaluating data assumes that leachate composition is controlled by the kinetics of the reactions that occur in the test vessel. Theoretically this is also the intended purpose of these tests, as implied by their names, even if they are not always applied as such.

The author generally supports the second method of assessment, unless the first method can be shown to produce reliable results. For this reason the application of humidity cells is favoured above kinetic leaching columns or other methods, as the microclimate in humidity cells is more easily controlled than through the use of other methods.

The kinetic approach, however, requires a more in-depth understanding of the processes that affect reaction rates, along with an understanding of geochemical controls as well. Depending on the test setup, corrections may be necessary for differences in temperature, moisture, reactive surface area and potentially even reaction rates. This approach is, however, necessary if reaction kinetics, rather than secondary minerals are the primary controls on water quality.

\section{$5 \quad$ When should AMD be assessed?}

The assessment of AMD is generally an iterative process and generally follows a risk based approach. This is complimented by a similar approach to optimising AMD management over the mine life. This philosophy, along with the real or perceived risk of AMD associated with mining operations, indicates that AMD should be assessed as early as possible in a mining project.

It is becoming general practice for AMD assessments to commence at pre-feasibility stage for most mining projects. Initially this may take the form of a desktop review of available geochemical and geological data for an exploration site. This approach has many benefits, including:

- an early indication of potential waste management or water quality issues that may be associated with a project

- an indication of possible mine planning strategies that may need to be implemented to minimise environmental risk

- an indication of environmental costs that may accrue to a project.

After pre-feasibility, AMD assessments generally follow an evolutionary path to characterise all lithologies in detail and to evaluate the physical environment in which they will occur. If this process is carried out effectively the data is useful for holistic mine planning, with a view to minimising environmental management costs during operations, as well as reducing long-term environmental liabilities post-closure. This is achieved by applying strategies to prevent or limit contaminant generation and contaminant transport to isolate potential contaminants from potential receptors (points of impact).

During operations, further refinement of AMD assessment and management strategies may be necessary as mine plans or material-handling strategies change, or improved methodologies or technologies are 
implemented. Operational management should, however, generally only be an iterative refinement of a carefully pre-planned material management strategy, if sufficient information is available to assess a proposed mining operation before operations commence. Operational management should be supported by a suitable monitoring programme to verify expected (modelled) system behaviour, as predicted prior to mining. If discrepancies are observed it may be necessary to alter management practices accordingly.

Upon closure most AMD management practices should already be established, although various remediation and treatments may be needed to prevent long-term impacts from closed mining operations. Continued monitoring of relevant parameters should occur until such time that a system is geochemically and physically stable, with due regard to long-term impacts. This may require a much longer term view than the 100 year view that has traditionally been applied to evaluate AMD, depending on the nature and location of the mine or mine infrastructure of interest.

\section{Where is AMD a concern?}

AMD can occur in various environments, although it is primarily associated with mining or quarrying, the main interest of this paper.

Other environments where acid drainage occurs (as a result of sulphide mineral oxidation) include coastal sediments. In these sediments iron sulphides are formed through bacterial reduction of sulphate and iron in contact with organic matter. Such soils are often referred to as acid sulphate soils (ASS). If these sediments are exposed to oxygen through dewatering, excavation or dredging, then the results are the same; acid is generated and elements that are potentially detrimental to the environment are mobilised. Some of the techniques used in the assessment and management of such sediments are transferrable to mine related AMD and vice versa.

As discussed previously in this paper the definition of AMD has become much broader than the processes associated with sulphide mineral oxidation and includes various other processes as well.

Primarily any metalliferous mining operation should be suspected of possessing the potential for AMD generation until proven otherwise. Mining operations often commence in weathered materials and subsequently progress into sulphidic materials. Some operations that only target oxidised materials encounter unexpected transitional (partially oxidised) or sulphidic materials. Additionally sulphide minerals may not occur in the ore zone, but may be associated with waste materials or wall rocks, e.g. some of the iron pits of Western Australia.

Sulphide minerals can also be problematic in quarrying operations and can result in unexpected waste management and water quality issues, as well as poor product quality.

Mining operations that are not associated with sulphide minerals can also produce potentially leachable materials and consequently leachates that are also classified as AMD according to modern usage. Such operations may include various oxidised mineral assemblages where mobile oxy-anion forming elements can accumulate, particularly in carbonate, phosphate, sulphate and chloride hosted mineral deposits. Reactive or leachable silicate minerals may also act as contaminant sources in some circumstances.

Considering the possible sources of AMD and the variety of environments in which it may occur, it is advisable that any large-scale excavation should be evaluated at least at a conceptual level by a suitably qualified person and that a limited number of screening tests should be carried out on targeted materials to eliminate or confirm the presence of AMD.

\section{$7 \quad$ Who should assess AMD?}

AMD assessments are carried out by people with a widely variable number of qualifications, with variable levels of regulation in different countries. Typically practitioners may include geochemists, metallurgists, chemical engineers, environmental engineers, hydrogeologists, soil scientists and environmental chemists. In each case it is possible for the person in question to provide a competent assessment, however, it is not uncommon for the AMD risk associated with a mineral deposit to be classified purely on the strength of ABA classifications and limited interpretation. In Canada it is a requirement for a registered Earth Scientist with a minimum level of experience to carry out an assessment, according to the NI 43-101 mining 
disclosure standard. In other countries various levels of professional registration are required, although they are not always enforced. Some professionals also lack skills in one or more aspects that are fundamental to understanding and evaluating AMD effectively. Important skills associated with AMD assessments include:

- adequate training in geology and mineralogy to understand the geochemical and physical characteristics of a mineral deposit or associated material

- sufficient geochemical knowledge to understand the origins and behaviour of potential contaminants in a mineral deposit

- sufficient chemical/metallurgical background to understand processing methodologies and how they affect the composition of mine wastes and effluents

- adequate training in data processing, modelling and statistics to quantify various aspects of a project appropriately

- an understanding of physical processes that control contaminant generation and contaminant transport. These include gas transport (e.g. oxygen transport to sulphide minerals), water transport (saturated, unsaturated and surface flow processes) along with solids transport (erosion processes).

Although not essential for a screening level assessment, knowledge of impact mechanisms and mitigation options is also required for higher level assessments.

From the recommended skills set, it is apparent that all the above listed professions may be capable of providing professional advice on assessment of AMD as each of them are trained in fields relevant to assessing and mitigating AMD. All of these professions will, however, typically be required to supplement their core skills with additional fields of expertise to achieve a holistic understanding of AMD. Effective AMD assessments often require the involvement of teams of people with the requisite skills between them and rarely individuals are sufficiently skilled in all relevant aspects. A high level of peer review is, however, still required as the same mistakes can be repeated over and over again if not corrected. Additionally each mine site is different, thus requiring the development of a complex conceptual model to understand cause and effect linkages. Adequate review is thus required to minimise the risk that important aspects of a mine conceptual model may be omitted or misrepresented. Historical failings associated with water quality prediction at mines in the United States were for instance highlighted by Kuipers et al. (2006). Although the situation continues to improve, the state of the art and reliability of AMD assessments is still being refined.

\section{Conclusion}

The philosophical and technical rationale associated with AMD assessments has been summarised briefly in this paper. It has been shown that AMD has become the main focus of environmental risk assessments associated with mining projects through a convergence of different factors. The question of AMD risk is a concern from various perspectives to a number of different stakeholders. Financial considerations are important to financiers, mining proponents and government and environmental implications to regulators and society in general.

At a technical level it has been shown that various aspects of a mining operation should be assessed and that this should occur from the early stages of ore reserve evaluation, continuing throughout the mine life and post closure.

The methodologies that are used to evaluate AMD potential can vary and their application to field conditions require a number of diverse technical skills. It is thus important for practitioners to be suitably trained and for them to be capable of exercising an adequate level of informed professional judgement for any mine site, being mindful of the methodologies they use.

\section{References}

AMIRA International Limited (AMIRA) (2002) ARD Test Handbook, Project P387A Prediction \& Kinetic Control of Acid Mine Drainage, AMIRA International Limited, Melbourne, Australia. 
Department of Industry Tourism and Resources (DoITR) (2007) Managing Acid and Metalliferous Drainage, Leading Practice Sustainable Development Program For the Mining Industry, Department of Communications, Information Technology and the Arts, Canberra, Australia.

Global Acid Rock Drainage (GARD) Guide (2009) International Network for Acid Prevention (INAP), www.gardguide.com.

Kirschner, L.A. and Grandy, E.B. (2003) Mining and the Vanishing Surety Bond Market, Parsons, Behle \& Latimer.

Kuipers, J.R., Maest, A.S., MacHardy, K.A. and Lawson, G. (2006) Comparison of Predicted and Actual Water Quality at Hard Rock Mines, The reliability of predictions in Environmental Impact Statements, EARTHWORKS, Washington DC, USA.

Price, W.A. (1997) Draft Guidelines and Recommendations for the Prediction of Metal Leaching and Acid Rock Drainage at Minesites in British Columbia, Reclamation Section, Energy and Minerals Division, Ministry of Employment and Investment, Smithers, British Columbia.

Price, W.A. (2009) Prediction Manual for Drainage Chemistry from Sulphidic Geologic Materials, MEND Report 1.20.1. CANMET Mining and Mineral Sciences Laboratories, Smithers, British Columbia.

Sobek, A.A., Schuller, W.A., Freeman, J.R. and Smith, R.M. (1978) Field and Laboratory Methods Applicable to Overburdens and Minesoils, Report EPA-600/2-78-054, U.S. National Technical Information Report PB-280 495. 